\title{
س
}

$>\mathrm{DE}$

$\cong$ PÉDAGOGIE

\author{
Recherches en éducation
}

172 | juillet-septembre 2010

La pédagogie universitaire : un courant en plein développement

\section{AgulHon Catherine \& XAVIER DE BRITO Angela. Les étudiants étrangers à Paris. Entre affiliation et repli}

Paris : L'Harmattan, 2009, 284 p.

\section{Valérie Erlich}

\section{(2) OpenEdition}

Édition électronique

URL : http://journals.openedition.org/rfp/2307

DOI : $10.4000 / \mathrm{rfp} .2307$

ISSN : 2105-2913

Éditeur

ENS Éditions

Édition imprimée

Date de publication : 15 juillet 2010

Pagination : 131-147

ISBN : 978-2-7342-1188-4

ISSN : 0556-7807

Référence électronique

Valérie Erlich, « Agulhon Catherine \& xavier De brito Angela. Les étudiants étrangers à Paris. Entre

affiliation et repli », Revue française de pédagogie [En ligne], 172 I juillet-septembre 2010, mis en ligne le 24 janvier 2011, consulté le 10 décembre 2020. URL : http://journals.openedition.org/rfp/2307 ; DOI https://doi.org/10.4000/rfp.2307

Ce document a été généré automatiquement le 10 décembre 2020.

(c) tous droits réservés 


\title{
AGULHON Catherine \& XAVIER DE BRITO Angela. Les étudiants étrangers à Paris. Entre affiliation et repli
}

Paris : L'Harmattan, 2009, $284 \mathrm{p}$.

\author{
Valérie Erlich
}

\section{RÉFÉRENCE}

AGULHON Catherine \& XAVIER DE BRITO Angela. Les étudiants étrangers à Paris. Entre

affiliation et repli. Paris : L'Harmattan, 2009, 284 p.

1 Cet ouvrage, composé de textes réunis et présentés par Catherine Agulhon et par Angela Xavier de Brito, traite des expériences individuelles et collectives des étudiants étrangers en France. Le processus de mobilité n'y est pas analysé à partir d'une vision centrée sur une problématique de l'internationalisation de l'enseignement supérieur, mais à partir d'une problématique de l'intégration sociale et culturelle. L'hypothèse posée est la suivante : les étudiants étrangers expatriés en France s'affilient à la culture d'accueil ou se replient sur leur culture d'origine lors de leur voyage d'études. Elle est interrogée par différents auteurs, doctorants pour la plupart qui ont, chacun de leur côté, mené une enquête qualitative auprès d'une centaine de jeunes venus étudier en France, issus de six zones géographiques distinctes (Brésil, Argentine, Chine, Maghreb, Afrique noire, Europe de l'Est). De ces travaux, présentés successivement dans six chapitres différents, on retiendra, à partir d'une lecture transversale, plusieurs conclusions, qui confirment ou approfondissent les analyses déjà produites sur les étudiants étrangers en France.

2 Tout d'abord, les étudiants étrangers apparaissent différents des migrants ordinaires, par leurs origines sociales et scolaires, par leurs aspirations, leurs projets de migration plus ou moins temporaires et leurs modes d'intégration institutionnelle. La migration étudiante est a priori non définitive et s'inscrit dans ce qu'on appelle aujourd'hui la 
mobilité internationale. Cependant la question du retour ou non au pays reste posée puisque certaines mobilités estudiantines (notamment chinoises et africaines) entraînent une fuite des cerveaux ou ce que l'on dénomme plus récemment une circulation des savoirs. En même temps, les enquêtes sur les étudiants étrangers montrent qu'un «habitus de migrant» (Angela Xavier de Brito, p. 19) les caractérise également puisqu'ils rencontrent des sentiments fréquents de domination, d'infériorité, de repli identitaire et des difficultés économiques proches de ceux des migrants ordinaires.

3 Le choix de la France repose sur des critères communs à la plupart des étudiants en mobilité : qualité de l'enseignement, prestige académique, coût des études, influence de certains professeurs, amis et relations (notamment pour les Argentins) sont les principaux. Il faut également ajouter l'attrait pour la capitale parisienne puisque l'ouvrage ne concerne que les étudiants en mobilité à Paris. La France n'est cependant pas toujours le premier choix des étudiants étrangers (les étudiants chinois lui préférant souvent les États-Unis). L'arrivée en France est décrite comme un processus d'affiliation (Coulon, 2005): les tracasseries administratives sont dénoncées par de nombreux étudiants de toutes origines (plus encore par les étudiants chinois et africains) ; la recherche d'un logement reste un défi difficile à dépasser ; l'exercice d'un travail rémunéré contraint le temps de travail studieux. Le processus d'acculturation concerne également les codes culturels, les valeurs, les habitudes, les façons de se comporter, différents de ceux des pays d'origine. L'apprentissage d'un habitus académique universitaire français (méthodes de travail, modes d'évaluations, distances aux enseignants...) est décrit comme déstabilisant par la plupart des étudiants en mobilité. L'appréhension de la langue française reste aussi un obstacle, en particulier pour les étudiants chinois. Le processus de «conversion-transformation» (Catherine Agulhon, p.137), dans lequel les étudiants étrangers sont engagés, relève du métissage et pour certains de l'assimilation. Il reste cependant moins traumatisant pour les étudiants issus des pays d'Europe de l'Est, dont la culture et les codes culturels sont plus proches de ceux de la France.

$4 \quad$ L'adaptation sociale est facilitée par le contact régulier avec des autochtones - petit(e) ami(e) français(e), logement en cité universitaire - mais le réseau de relations des étudiants étrangers reste lié le plus souvent au principe de "similarité attraction » : le partage d'une même langue et de codes culturels similaires rapprochant les individus, le mixage social reste faible; les étudiants chinois "vivent entre eux ", les étudiantes africaines « se replient sur leur réseau national »... D’une manière générale, les travaux présentés montrent une opposition entre les «privilégiés " (élite internationale) et les "précarisés", entre les "boursiers" et les «non boursiers", qui ne se côtoient pas même lorsqu'ils sont accueillis dans les mêmes institutions universitaires et dont les rythmes de vie diffèrent radicalement. Cette séparation est particulièrement marquée entre les étudiants chinois. L'élite internationale est décrite à travers l'expérience des étudiants chinois dans les grandes écoles, soutenus par les gouvernements, inscrits dans le cadre de programmes d'excellence, ce qui leur assure des conditions de vie et d'études privilégiées, contrairement aux étudiants chinois "privés» venus par leurs propres moyens.

5 Les auteurs constatent enfin la fabrication d'un « capital international » : il se construit à travers la familiarité qu'ont certains étudiants à partir de leurs propres expériences de déplacements antérieures (lycée international, déplacements personnels), à travers 
leur familiarité avec l'étranger (passé de migration, contacts avec des personnes qui ont voyagé ou vécu à l'étranger), ce qui caractérise plus les étudiants brésiliens et argentins ainsi que les étudiants polonais que ceux d'autres nationalités. Ce capital favorise nettement l'adaptation socioculturelle en France mais également le capital social acquis en France.

6 Reposant sur une enquête qualitative auprès d'une centaine d'individus, cette recherche permet finalement d'explorer les modes d'affiliation scolaire et culturelle des étudiants en mobilité. Elle ne peut cependant prétendre à l'exhaustivité. Son objectif reste modeste. Complémentaire à d'autres études, elle confirme les analyses quantitatives récentes (Ennafaa \& Paivandi, 2008) produites sur les conditions d'intégration des étudiants par nationalité et qui marquent bien l'impact de l'origine géographique. Un tableau synoptique des modes d'affiliation de ces jeunes selon leurs origines aurait d'ailleurs été le bienvenu, afin de faciliter la lecture transversale des études présentées dans l'ouvrage. Le parti pris d'une approche résolument qualitative permet de dépasser cependant l'analyse causale en cherchant à révéler le sens que les migrants pour études accordent à leur expérience en France. Cette approche nous rappelle également que, malgré les politiques d'immigration choisie, mises en place ces dernières années pour réguler les flux d'étudiants en mobilité, la figure de l'étudiant étranger, venu étudier librement en France et dont le devenir n'est pas encore défini, n'a pas disparu au seul profit de l'étudiant international, étudiant compétitif bénéficiant de programmes gouvernementaux et représentant l'«élite migratoire " (Endrizzi, 2010). La fin de l'étudiant étranger (Slama, 1999) ne serait donc qu'une fiction institutionnelle. Le mérite de cet ouvrage est aussi de nous rappeler que ces étudiants «boursiers» et "non boursiers" qui s'opposent sur bien des plans (économiques, sociaux, culturels...) partagent pourtant des difficultés d'affiliation semblables. Les logiques politiques et internationales ne justifient pas non plus toutes les stratégies et capacités d'adaptation des migrants pour études.

7 C'est donc bien la question de l'intégration des étudiants étrangers en milieu parisien qui est posée dans cet ouvrage. La typologie empruntée à Dominique Schnapper (2007) permet aux auteurs de repérer quatre ferments d'intégration : sur un plan structurel, l'intégration est réussie puisque les étudiants en mobilité s'intègrent aux institutions dans le pays d'accueil ; l'intégration sociale est plus souvent caractérisée par le repli que par l'ouverture; l'intégration culturelle est double, partagée entre les valeurs et modes de pensée des pays d'accueil et d'origine; enfin sur le plan identitaire, l'intégration reste inachevée. De ces conclusions globalisantes, ressortent des différenciations individuelles, également au sein d'une même nationalité d'origine, liées aux histoires familiales, scolaires et de migration de ces jeunes en mobilité, qui amènent à penser que l'intégration reste un mythe lié à la problématique de la migration en France. Les auteurs précisent d'ailleurs dès l'introduction de l'ouvrage que les questions d'intégration, "idéologiques et le plus souvent traitées de manière idéologique ", restent cependant à l'ordre du jour. Ces questions au cœur de la sociologie de l'immigration nourrissent de nombreuses controverses, mais on peut cependant regretter que les notions d'identité, d'étiquetage, de stigmatisation ne soient pas abordées de manière plus approfondie à travers ces différentes études, alors même que les coordonnateurs de l'ouvrage rappellent que « les recherches sur la construction de l'identité complètent avantageusement les approches en termes d'intégration ». Il aurait été intéressant en particulier de développer les analyses concernant la recomposition des identités collectives de ces jeunes migrants en France, en essayant 
de mieux montrer comment ils reconstruisent, en situation de mobilité, leur identité sociale, scolaire et familiale en France, tout en étant tiraillés entre leur culture d'accueil et leur culture d'origine. Ceci afin d'approfondir les réponses à la question posée en préface de l'ouvrage par Éric Plaisance : « Peut-on parler d'intégration comme transformation identitaire?»

\section{BIBLIOGRAPHIE}

COULON A. (2005). Le métier d'étudiant. L'entrée dans la vie universitaire. Paris : Economica.

ENDRIZZI L. (2010). « La mobilité étudiante, entre mythe et réalité ». Dossier d'actualité de la VST, $\mathrm{n}^{\circ}$ 51. Disponible sur Internet à l'adresse : <http://www.inrp.fr/vst/LettreVST/51-

fevrier-2010.php> (consulté le 15 juillet 2010).

ENNAFAA R. \& PAIVANDI S. (2008). Les étudiants étrangers en France. Paris : La Documentation française.

SCHNAPPER D. (2007). Qu'est-ce que l'intégration? Paris : Gallimard.

SLAMA S. (1999). La fin de l'étudiant étranger. Paris : L'Harmattan.

\section{AUTEURS}

VALÉRIE ERLICH

Migrations et société, université de Nice-Sophia-Antipolis 